\title{
INFLATION IN EINSTEIN-CARTAN THEORY WITH \\ ENERGY-MOMENTUM TENSOR WITH SPIN
}

$$
\text { by }
$$
A. J. Pennelly (a)

\author{
MS-47 Teledyae Browa Engineering \\ Cummings Research Park \\ Buntsville, AL 35807
}

\begin{abstract}
James C. Bradas (a)
AMSMI-RD-GC-T, Research Development and Engineering Center,

U.S. Arwy Missile Command,

Redstone Arsenal, AL 35898
\end{abstract}

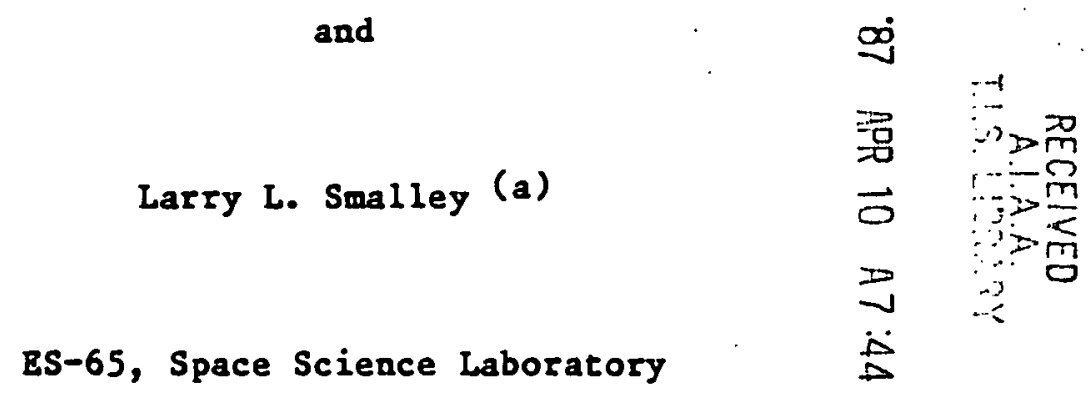

Marshall Flight Center, AL 35812

(NASA-TM-101164) INFIATICN IN

EINSTEIN-CARTAN TEEORY WITE FLEEG-EOUENTDE

N $88-24547$

TENSOE WITH SEIL (NASA) $18 \mathrm{~F}$ CSCL 20C 


\section{Abstract}

Generalized, or "power-law," inflation is shown to necessarily exist for a simple, anisotropic, (Bianchi Type-I) cosmology in the Einsten-Cartan gravitational theory with the Ray-Smalley improved energy-momentum tensor with spin. Formal solution of the EC field equations with the fluid equations of motion explicity shows inflation caused by the RS spin angular kinetic energy density.

(a) Also with Department of Physics, Oniversity of Alabama in Buntsville, AL 35899. 
INFLATION IN EINSTEIN-CARTAN THEORY WITH IMPROVED ENERGY-MOMENTUM TENSOR WITH SPIN

\title{
by
}

\author{
A. J. Fennelly, James C. Bradas and Larry L. Smalley
}

Introduction. The early universe is expected to undergo a period of inflation (exponential expansion) due to the presence of bare quantum fields which behave like a cosmological constant term in the field equations. That period of accelerated expansion can also exist as a state of "power-law inflation" in which the expansion scale factor is a power-law function of the time. ${ }^{2}$ In either case, $R \sim e^{R t}$ of $R \sim t^{N}$, inflation, is important because it may offer a simultaneous solution to the cosmological problems of horizons, homogeneity, and flatness. 2

Inflation may also provide a solution to the origin of the presently observed large-scale isotropy of the university. 3 Its presence minus the acceleration which would be produced by a now zero cosmological constant, which is quite effective in isotropizing the universe. 4 There is, however, the not fully resolved question of whether inflation occurs, and is effective in producing isotropy, if the initial shear of spatial curvature of the universe is sufficiently large. 5 One would therefore like to find models with inflation, even power-law inflation, that possess inevitable inflation no matter how large the shear. 
Power-law inflation has been invoked to explain the structure of inhomogeneity in the universe.3,6 It has been discussed as the result of particle-creation processes in the early vacuum dominated universe, 7 the result of evolution and compactification of certain models of Raluza-Rlein cosmology, 8 the consequence of the antisymetric part of the affine connection in the nonsymetric theory of gravity of Moffat, 9 and the effect of a root-mean square spin energy densitglo in the Einstein-Cartan theory with the Ray-Smalley improved energy-momentum tensor with spin.11 In this paper, we show that a generalized (or power-law) inflationary (and super-inflationary) epoch arises naturally and inevitably in the early universe in a cosmological model in the Einstein-Cartan gravitation theory with the improved energy-momentum tensor with spin of Ray and Smalley.

Torsion, Spin Energy Density, and the Ray-Smalley Improved Energy-Momentum Tensor With Spin. Spin is, a Lorentz-invariant quantum mechanical phenomenon. This means that it exists in flat space and as such represents rotation with respect to nothing. It must, therefore, properly be included in the definition of internal energy of a fluid.12 Furthermore, rotation itself can have a variety of forms, even if we are considering only rotating coordinate systems and references frames and not rotating matter with or without internal spin.13 The former requries a reformulation of the fluid lagrangian and the first law of thermodynamics to include the angular kinetic energy density of spin in determination of the equations of motion and field equations.14 The latter requires that torsion be included in any spacetime formulation of physics that includes an affine connection. 15 
In a further convincing argument, Penrose has shown that torsion naturally arises when the general class of conformal transformations of metrics is allowed to include complex transformations.16 This occurs if one wishes to preserve the Lorentz-invariant two - component spinor. calculus in as natural a form as possible. With a conformal transformation $g_{a b}=\Omega \bar{\Omega}$ gab for complex $\Omega$, the torsion is generated as $T_{a b} c=i\left(\nabla_{d} \log \Omega-\nabla_{d} \log \bar{\Omega}\right) E_{a b} c d$, where $i=\sqrt{-1}$ and $E_{a b}{ }^{c d}$ is the four-dimensional alternating symbol.

Thus, we are directed to the Ray-Smalley formalism for the Binstein-Cartan theory with improved energy-momentum tensor with spin. The internal energy $\varepsilon$ of a fluid with spin is then given by

$$
d \varepsilon=T d s-\operatorname{Pd}(T / \rho)+\frac{1}{2} w_{i j} d s^{i j}
$$

$\because \ldots . .$.

here $T, S, P, \rho, w_{i j}$ and $s^{i j}$ are the fluid's temperature, specific entropy, pressure, density of inertia, spin angular velocity, and specific spin angular momentum. The spin angular velocity $W_{i j}$ is defined by

$$
w_{i j}=\dot{a}_{i}^{\gamma} a_{\gamma j}=a_{j}^{\gamma} a_{\gamma j / / k^{U}}
$$

where $a v_{i}, u_{k}$, and $/ / k$ are the tetrad vectors, four-velocity of the fluid, and the covariant derivative. The spin density $s_{i j}$ of the fluid is given by

$$
s_{i j}=\rho K\left(a^{1}, a_{j}^{2}-a_{j}^{1} a_{i}^{2}\right)=\rho s_{i j}
$$


where $K$ is a coupling constant. The two combine to form the spin kinetic energy density $T$ of the fluid:

$$
T=\frac{1}{2} s_{1 j} w^{1 j}
$$

One choice that can be made for the tetrad vectors is to identify one of them with the fluid four velocity: $\sigma^{i}=a^{4 i}$.

A lagrangian has been given for this model by Ray and Smalleyll but is not necessary here. Appropriate variations lead to equations of motion and field equations. First of these is the equation of motion for the spin:

$$
\operatorname{DS}_{1 j}=2 \mathrm{w}_{\ell}\left(i \mathrm{~S}_{j}\right)^{\ell}
$$

where $D$ is the total convective derivative.. The field equation is . - :

$$
G^{l K}-\ddot{\nabla}_{l}\left(R^{l i R}+R^{i R l}\right)=T_{f}^{1 k}+T_{s}^{1 k}
$$

where the modified torsion tensor is

$$
K_{i j}^{K}=T_{1 j} k+2 \delta^{K}\left[1^{T} j\right] l^{l}
$$

and the square brackets denote antisymetrization. The perfect fluid part of the energy-momentum tensor $T_{F}^{1 k}$ is:

$$
T_{F}{ }^{1 K}=\rho(1+\varepsilon+P / \rho) U^{i} U^{K}+g^{i k}
$$

and its intrinsic spin part $T_{s}{ }^{i k}$ is: 


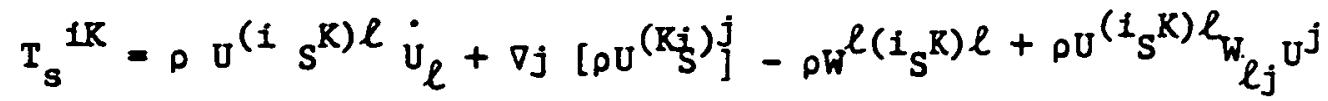

where the last two terms arise from the inclusion of spin as a thermodynamic variable in the first law of thermodynamics. The torsion is detemined from the spin density tensor by

$$
R_{i j} R=s_{i j} R
$$

where $S_{i j} k$ is the canonical spin-density tensor of Halbwachs:11

$$
s_{i j} R=s_{i j} U^{R}
$$

The antisymetric field equation is

$$
\left(\nabla_{1}-T_{i j}^{j}\right) s_{k l}^{i}=2 T_{[K l]}
$$

and is mainly an expression for the conservation of angular momentum. The above equations constitute the Einstein-Cartan theory with the RaySmalley energy-momentum tensor.

A notable success of the theorty has been the solution for a static cylinder with perfectly aligned spins of its constituent particles, found by Tsoubelis.17 It is a significant improvement, in that consistent exterior-interior matching conditions are fulfilled, and that the exterior gravitational field possess off-diagonal terms in the 
metric which cannot be transformed away. This indicates that a true magnetic-trype gravitational field is generated by spin in the improved EC with RS energy-mometum tensor with spin.

Cosmological Models in Gravitation Theories with Torsion. A large number of cosmological models have been exhibited in EinsteinCartan theories. However, we shall mention only a representative few of them, of direct relevance here. The most recent study of homogeneous and isotropic cosmological models in torsion gravity theories with the standard energy-momentum tensor has been given by Goenner and MullerHoissen for lagrangian densities containing terms in $\mathrm{T}^{2}$ and $\mathrm{R}^{2}$, a more general gravitational lagrangicis than what we consider later.18 Contributions to the field equations arising from the $T^{2}$ and $R^{2}$ terms will also drive an inflationary epoch, 19 beyond the contribution of the spin kinetic energy density terms shown here.

Anisotropic cosmologies for BC theory with the standard lagrangian and standard energy - momentum tensor have been studied in an interesting manner by Tsoubelis and by Lorenz.21-23 Those represent the clearest treatment of anisotropic cosmologies with torsion for Bianchi Types $V I_{0}$ and $V I I_{0}, 20$ Type $\nabla, 20,24$ Rantowski-Sachs geometries, 22 and a general class of Bianchi types with magnetic field.21

There have been some recent limited studies of cosmological models in EC theory with the improved RS energy-momentum tensor with spin. These have mostly concentrated on the Godel solution or Godellike cosmologies.24-25 Their particular success has been in providing reasonable perfect-fluid sources for the Godel models but with, furthermore, causal behavior of the geodesics. 
In expanding models, there have been very few investigations published thus far using the RS energy-momentum tensor. One was a consideration of the role of spin, with the energy-momentum tensor with spin but not the improved energy-momentum tensor with spin.26 While we -do not completely agree with all the details of their calcuations, we believe their conclusion is accurate. Their computation shows that internal spin drives expansion of a fluid positively.

Gasperini has demonstrated that the improved RS energy-momentum tensor with spin may drive inflation.10 It is proven approximately and ad hoc for a flat Robertson-Walker (homogeneous and isotropic) cosmological model. Much of the argument is unsatisfactory in that lacks rigor, but the main conclusion has a certain validity. Our result, which confirms both that of Bedran and Vasconcellos-Vaidya26 and that of Gasperini,10 shows that such approximations are unnecessary to demonstrate that inflation is inevitable if the universe is correctly. described by the EC theory with the improved RS energy-momentum tensor. Inevitable Inflation in a simple Anisotropic Einstein Cartan/Ray-Smalley Cosmological Model. We will now examine a simple anisotropic model to demonstrate our result. The spatial geometry is Bianchi Type I, indicating we consider only the effects of shear (dynamic anisotropy) and not of curvature (kinematic or static anisotropy). The anisotropy is necessary so that global spin angular kinetic energy effects can be examined without resorting to the averaging arguments and approximations necessary in the Roberton-Walker mode 1.10 
We choose the metric to have the form-first introduced by Misner: 27

$$
d s^{2}=-d t^{2}+e^{2 \alpha} e^{2 B} i j d x^{i} d x^{j},
$$

where $e^{2 \alpha}=R(t)$ the scale factor, and $B_{i j}$ is a $3 \times 3$ symetric, tracefree, matrix. We use the method of differential forms and so write

$$
d s^{2}=g_{\alpha \beta} w^{\alpha} w^{\beta}
$$

where we take $\varepsilon_{\alpha} B$ to be the Lorentz metric and $w^{a}$ to be a basis of differential forms.28 We then have that wo $=d t$ and $w^{i}=e^{\alpha} e_{i j}^{B} d x^{j}$. To show more clearly how torsion is inserted in the connection forms, we rewrite the first Cartan equation in the form $\therefore:$

$$
W_{\gamma}^{\mu} \wedge W \gamma=\frac{1}{2} T_{\lambda \gamma}: \mu w^{\lambda} \cdot w^{\lambda}-d w^{\mu},
$$

where ${ }^{w} \gamma$ are the connexion forms and $d$ indicates the exterior derivative operation on a form. The dual to the basis are the tetrad vectors themselves and they obey the usual orthonormality relations $q_{\mu} i_{a_{\gamma}}=g_{\mu \gamma}$ and $a_{\mu i} a \mu_{j}=n_{i j}$ where $\eta_{i j}$ signifies the usual Lorentz metric. Using tetrads consistent with the choice of Bianchi Type I, we take the nonzero components of $s_{i j}$ to be

$$
s_{12}=K(x)
$$

where $K^{(X)}$ is some general function. The tracefree proper torsion $\mathbb{R}_{i j}^{K}$ is related to the spin density by $\bar{K}_{i j}{ }^{K}=\rho s_{i j}$ UK. 
Proceeding with the usual calculations, including use of the second Cartan equation, we find the usual relativistic quantities and equations: The affine connexion forms are

$$
w_{j}^{o}=\left(\dot{\alpha} \delta_{j k}+\sigma^{j k}+\frac{1}{2} T_{j k}^{o}\right) w^{K}
$$

and

$$
w j_{k}=-\tau j k d t \text {, where } \sigma j k \text { and } \tau j k \text { are the symetric and }
$$
antisymetric parts of

$$
\left(e^{B}\right)_{j \mathrm{j}} \quad\left(e^{-B}\right)_{\mathrm{K}} \ell
$$

and the overdot indicates the time derivative. Reeping maximm simplicity, we choose a comoving fluid with four-velocity $u_{a}=\gamma_{\alpha}^{0}$, $u^{a}=\delta_{0^{\circ}}^{a}$ The Einstein-Cartan equations can be put into the form

$$
\begin{aligned}
& G_{o 0}=3 \dot{\alpha}^{2}-\frac{1}{2} \sigma_{i j} \sigma^{i j}=\rho(1+\varepsilon) \\
& G_{R}^{K}=\ddot{6 a}-9 \dot{\alpha}^{2}-\frac{3}{2} \sigma_{1 j} \sigma^{i j}=3 p+2 \rho \tau_{i j} s^{j 1}
\end{aligned}
$$

$$
\text { is }
$$

and

$$
\begin{gathered}
G_{i j}-\frac{1}{3} \delta_{i j} G_{R}^{K}=\dot{\sigma}_{i j}+3 \dot{a} \sigma_{i j}+[\sigma, \tau]_{i j}-\frac{1}{2}\left[\dot{T}_{i j}^{0}+3 \dot{\alpha T_{i j}}{ }^{o}\right]-\frac{1}{2} T_{i K}{ }^{o} \tau_{j}^{K} \\
\quad=T_{i j}-\frac{1}{3} \delta_{i j} T_{K}^{K}=\frac{1}{2} \rho \tau_{K j} s^{i K}
\end{gathered}
$$


with $G_{0 i}=T_{0 i}=0$. It seems as though the $G_{00}=T_{00}$ equation has no spin kinetic energy terms, but one should recall that the term $\varepsilon$ contains spin kinetic energy terms via Eq. (1). Indeed we could approximately write

$$
\varepsilon=T S-P / \rho+\frac{1}{2} W_{i j} s^{i j}
$$

under certain circumstances. But it is true that under most circumstances the term in $\rho$ itself in the $T_{00}$ component will dominate.

Requirements of symetry and consistency give that

$$
\dot{\mathrm{T}}_{12}^{0}+3 \dot{a} \mathrm{~T}_{12}^{\circ}=0
$$

which solves to $T_{12^{\circ}}=I_{12^{\circ}}(0) e^{-3 \alpha}$, where $T_{12^{\circ}}(0)$ is an integration constant (initial value). We can absorb the $(1+\varepsilon)$ term into $p$ and manipulate and combine the Einstein-Cartan equations to find

$\ldots a \quad \ddot{a}=-\frac{1}{2} \sigma_{i j} \sigma^{i j}-\frac{1}{2}(\rho+P)+\frac{1}{3} \rho \tau_{i j} s^{1 j}$

Then substitution of $\ddot{a}=\ddot{R} / R-(\dot{R} / R)^{2}$

and $\dot{\alpha}=\dot{R} / R$ into Eq. (24) gives

$$
\ddot{R}=-\frac{2}{3} \rho_{\sigma}-\frac{\rho}{6}-\frac{P}{2}+\frac{\rho}{2} \tau_{i j} s^{i j}
$$

We must now determine the way each term on the right hand side of Eq. (10) scales with the volume expansion factor $R$. It is clear that while the terms for shear energy density $\rho_{\sigma}=\frac{1}{2} \sigma_{i j} \sigma^{i j}$, inertia density $\sigma$, 
and pressure $p$ are negative, the term for spin angular kinetic energy density contributes positively to $R$. One must see if it ever dominates. To examine that we need only establish the dominant scaling relation of each energy density with R, formally and to leading order.

The contracted Bianchi identity and E-C field equations combine to give the usual conservation equation for the inertia density, with equation of state $p=\gamma: \dot{\rho} / \rho+3 \dot{\alpha}(1+\gamma)=0$, with the solutions $\rho=\rho_{0} R^{-3(1+\gamma)}$. The shear evolution equations have the usual leading order formal solution which gives the scaling 1 aw $\rho_{\sigma}=\rho_{\sigma}^{0} R^{-6}$. The tensor $\tau_{i j}$ is identically the rotation rate of the observer's Fermitransported reference $\operatorname{triad}^{29}$ and so equals $\mathrm{w}_{i j}$ of Eq. (2). Dimensional analysis 30 shows that its evolution (from the EC equations) scales with proper time $t$ as $\tau_{i j}=\tau_{i j}^{0} t^{-1}$.

The scaling relation between $R$ and time $t$ is given from the $G_{00}=T_{00}$ field equation. Depending on which form of matter, or the shear, dominates; $\mathrm{R}-t^{1 / 3}$ for shear or stiff (scalar fields) matter $(q=1)$, and $R-t^{1 / 2}$ for radiation $(Y=1 / 3)$, and $R \sim t^{2 / 3}$ for dust or low-pressure matter $(\gamma=0)$. The scaling relation for the given density comes from the equations of motion above. That gives $s_{i j} \sim t^{-1}$. Combining those results for density, spin density, and tetrad rotation, we find that

$$
\rho_{i j}^{\tau} s^{i j}=\rho_{0} \tau^{0}{ }_{i j} s_{0}^{i j} R^{-3(3+\gamma)}
$$

Combining the above results into Eq. (11), we obtain the equations

$$
\dot{R}=\frac{-\rho}{3} R^{-2}-\frac{2}{3} \rho_{\sigma}^{0} R^{-5}+\frac{1}{3} \rho_{0} \tau_{i j}^{0} s_{i j}^{0} R^{-9}
$$


for dust,

$$
\ddot{R}=-\frac{\rho}{3} R^{-3}-\frac{2}{3} \rho_{\sigma}^{0} R^{-5}+\frac{1}{3} \rho_{0} \tau_{i j}^{0} S_{i j}^{0} R^{-10}
$$

for radiation, and

$$
\ddot{k}=-\frac{2}{3}\left(\rho_{0}+\rho_{\sigma}\right) R^{-5}+\frac{1}{3} \rho_{0} \tau_{i j}^{0} s_{i j}^{\circ} R^{-12}
$$

for stiff matter or scalar fields. In each of Eqs. (12), for sufficiently small values of $R$, the spin density term will dominate and thus an early epoch cosmology will have its expansion accelerated by the spin kinetic energy density of the fluid. The shear cannot effectively damp the acceleration for sufficiently early epochs.

Conclusion. Note that the important term in the equations is of the form $\tau_{i j} s^{i j}$ and so it doesn't matter what components of $\tau_{i j}$ and $s^{i j}$ are nonzero. For computational simplicity, we could choose only $\tau_{12} \neq 0$ and $s^{12} \neq 0$, and then $\sigma_{12}$ is the only off-diagonal component of $\sigma_{i j}$. We do find $s_{11}=\sigma_{22}$ from this (axial symetry) and the true fluid vorticity then turns out to be $\Omega_{12}=-T_{12}{ }^{0} 0_{0}$. In the future, we will extend this work to other Bianchi types (for curvature anisotropy) and magnetic and magnetohydrodynamic models (for matter anisotropies). The computation presented here at least shows that the EC theory with RS EMT provides us with cosmological models with inevitable inflation in the early universe, exhibiting a simple and natural explanation for the apparent inflationary epoch which may have occurred. 


\section{References}

1. A. D. Linde, Rep. Prog. Phys. 47, 925 (1984); R. Brandenbage, Rev. Mod. Phys. 57, 1 (1985).

2. L. J. Abbott and M. B. Wise, Nucl. Phys. B244, 541 (1984); Astrophys. J. 282, 447 (1984); Phys. Lett. 135B. 279 )1984).

3. 0. Gron, Phys. Rev. D32, 2522 (1985), 1584 (1985); D33, 1204 (1986); J. Math Phys. 27, 1490 (1986); J. Bllis and R. A. Olive, Nature 303, 679 (1983).

.4. R. Babbri, Lett. Nuovo. Com. 26, TF (1979); R. M. Wald, Phys. Rev. D28, 2118 (1983); B. Weber, J. Math Phys. 25, 3279 (1984).

5. F. Steigman and M. S. Turner, Phys. Lett. 128B, 295 (1983); T. Rothman and M. S. Madsen, ibid. 159B, 256 (1985); J. D. Barrow and M. S. Turner, Nature $\underline{292}, 35$ (1985); M. Demianski, Nature $\underline{307}, 140$ (1984); L. G. Jensen and J. A. Stein - Schabes, Phys. Rev. D34, 931 (1986).

6. B. L. Spokoiny, Phys. Lett. 147B, 39 (1984).

7. F. T. Lucchin and S. Matarrese, Phys. Rev. D32, 1316 (1985). 
8. R. B. Abbott, S. M. Barr, and S. D. Ellis, Phys. Rev. D30, 720 (1984); R. B. Abbott, S. D. Ellis, and S. M. Barr; ibid, D3s, 673 (1985).

9. J. W. Moffat and D. Vincent, Can. J. Phys. 63, 321 (1985).

10. M. Gasperini, Phys. Rev. Lett. 56. 2873 (1986).

11. J. R. Ray and L. L. Smalley, Phys. Rev. 26, 2619 (1982); 27, 1383 (1983); Phy. Rev. Lett. 49, 1059 (1982); see also Phys. Rev. D26, 2615 (1982).

12. L. D. Landau and B. M. Lifshitz, Statistical Physics (Pergamon, New York, 1980), PP. 74-76, Pp. 104-106.

13. B. Chaknaboity and S. Sarkar, Ann. Phy8. (N.Y.) 163, 167 (1985).

14. Approaches along these lines have recently been given in G. Cognola, R. Soldati, L. Vanzo, and S. Zerbini, Phys. Rev. D25, 3109 (1982); Nuovo Cim, 76B, 109 (1983); D.de Ritis, M. Lavorgna, G. Platania, and C. Stornaiolo, Phys. Lett. 95E, 425 (1983); Phys. Rev. D28, 713 (1983); ibid. 31D, 1854 (1985); R. Amorim, J. Math. Phys. 25, 874 (1984); Phys. Lett. 104A, 259 (1984); Gen. Rel. Grav. 17, 525 (1985); Phys. Rev. D31, 3099 (1985); ibid. 33D, 2796 (1986); W. Ropczynski, Phys. Rev. D34, 352 (1986); and see Ref. 11. 
15. R. J. Petti, Gen. Rel. Grav. I, 869 (1976); ibid. 18, 441 (1986); B. Gogala, ibid. 19, 573 (1980); ibid. 19,587 (1980); P. B. Yasskin, Gen. Rel. Grav. 13, 463 (1981).

16. R. Penrose, Found. Phys. 13, 325 (1983).

17. D. Tsoubelis, Phys. Rev. Lett. 512235 (1983); Phys. Rev. D30, 1632 (1984).

18. H. Goenner and F. Muller - Hoissen, Class. Quant. Grav. 1, 651 (1984).

19. B. Whitt, Phys. Lett. 45B, 176 (1984); and discussion S. W. Hawking and J. C. Luttrell, Nucl. Phys. B247, 250 (1984).

20. D. Tsoubelis, Phys. Rev. D20, 3004 (1979) N. A. Batakis and D. Tsoubelis, Phys. Rev. D26, 261 (1982).

21. D. Lorenz, Acta. Phys. Pol. B12, 939 (1981).

22. D. Lorenz, J. Phys. A., 15, 2809 (1982).

23. D. Lorenz, Gen. Rel. Grav. 14, 691 (1982).

24. E. P. Vasconcellos-Vaidya, M. L. Bedran, and M. M. Som, Prog. Theor. Phys. 22, 857 (1984); M. L. Bedran, E. P. VasconcellosVaidya, and M. M. Som, Nuovo Cim. 87B, 101 (1985). 
25. L. L. Smalley, Phys. Rev. D15, 3124 (1985).

26. M. L. Bedran, and E. P. Vasconcellos-Vaidya, Lett. Nuovo Cim, 41, 73 (1984).

27. C. H. Misner, Ap.J. 151, 431 (1968).

28. M. P. Ryan and L. C. Shepley, Howogenous Relatiristic Cosmologies, Princeton University Press, Princeton (1975).

29. A. J. Eennelly, Ap.J. 207693 (1970), ibid. 252, 410 (1982).

30. A. J. Fennelly, J. Math Phys. 22, 126 (1981). 